Antonio de Padua Albuquerque Oliveira

Engenharia de Requisitos Intencional: Um Método de Elicitação, Modelagem e Análise de Requisitos

Tese de Doutorado

Tese apresentada ao Programa de Pós-Graduação em Informática do Departamento de Informática da PUC-Rio como parte dos requisitos parciais para obtenção do título de Doutor em Ciências.

Orientadores: Julio Cesar Sampaio do Prado Leite Luiz Marcio Cysneiros

Rio de Janeiro

Março de 2008 
Antonio de Padua Albuquerque Oliveira

\section{Engenharia de Requisitos Intencional: Um Método de Elicitação, Modelagem e Análise de Requisitos}

Tese apresentada como requisito parcial para a obtenção do grau de Doutor pelo Programa de Pós-graduação em Informática do Departamento de Informática do Centro Técnico e Científico da PUC-Rio. Aprovada pela Comissão Examinadora abaixo assinada.

Prof. Julio Cesar Sampaio do Prado Leite

Orientador

Departamento de Informática - PUC-Rio

Prof. Luiz Marcio Cysneiros

Co-orientador

School of Information Technology - YorkU

Prof. Marco Antonio Casanova

Departamento de Informática - PUC-Rio

Profa. Simone Diniz Junqueira Barbosa

Departamento de Informática - PUC-Rio

Prof. Jaelson Freire Brelaz de Castro Centro de Informática - UFPE

Profa. Vera Maria Benjamim Werneck Departamento de Informática - UERJ

Prof. José Eugênio Leal Coordenador Setorial do Centro Técnico Científico - PUC-Rio

Rio de janeiro, 27 de Março de 2008 
Todos os direitos reservados. É proibida a reprodução total ou parcial do trabalho sem autorização da universidade, do autor e dos orientadores.

\section{Antonio de Padua Albuquerque Oliveira}

Graduou-se em Engenharia Elétrica na Universidade Federal do Rio de Janeiro (UFRJ) e obteve o grau de Bacharel em Ciências Estatísticas na Escola Nacional de Ciências Estatísticas (ENCE) em 1975. Obteve o título de Mestre em Informática na PUC-Rio em 1994. É professor assistente da Universidade do Estado do Rio de Janeiro (UERJ) desde 1996, onde leciona disciplinas na área de Engenharia de Software. É pesquisador na área de Engenharia de Software para Sistemas Multi-Agentes do Laboratório de Engenharia de Software (LES) da PUC-Rio.

Ficha Catalográfica

Oliveira, Antonio de Padua Albuquerque

Engenharia de requisitos intencional: um método de elicitação, modelagem e análise de requisitos / Antonio de Pádua Albuquerque Oliveira ; orientadores: Julio César Sampaio do Prado Leite, Luiz Marcio Cysneiros. - 2008.

$$
261 \mathrm{f} \text { : il. ; } 30 \mathrm{~cm}
$$

Tese (Doutorado em Informática) - Pontifícia Universidade Católica do Rio de Janeiro, Rio de Janeiro, 2008.

Inclui referências bibliográficas.

1. Informática - Teses. 2. Engenharia de requisitos orientada a metas. 3. Engenharia de requisitos. 4. Elicitação de requisitos. 5. Sistemas multi-agentes. 6. Agentes de software. 7. Engenharia de software. I. Leite, Julio Cesar Sampaio do Prado. II. Cysneiros, Luiz Marcio. III. Pontifícia Universidade Católica do Rio de Janeiro. Departamento de Informática. IV. Título. 


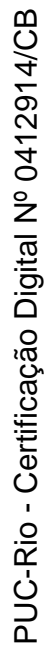

Aos meus pais José Agapito de Oliveira e Jovita Albuquerque de Oliveira. 


\section{Agradecimentos}

À Deus.

À minha família.

Ao professor Julio Cesar Sampaio do Prado Leite pelo apoio, pela confiança e oportunidades, pelo convívio acadêmico e pelo conhecimento transmitido.

Aos professores membros da banca por aceitarem o convite para a participação e por ajudarem o trabalho com criticas e contribuições.

Ao professor Ricardo Choren, suplente da banca, por aceitar o convite para a participação e por ajudar o trabalho com criticas e contribuições.

Aos amigos da PUC-Rio que fiz durante estes quatro anos de doutorado.

Aos amigos em Toronto, Canadá: Luiz Marcio Cysneiros, Radu Campeanu e Younes Benslimane que fiz durante um ano que estive como visitante na York University.

Ao LES da PUC-Rio

À UERJ e ao Departamento de Informática da PUC-Rio, pelos auxílios concedidos, sem os quais este trabalho não poderia ter sido concluído. 


\section{Resumo}

Oliveira, Antonio de Padua Albuquerque; Leite, Julio Cesar Sampaio do Prado; Cysneiros, Luiz Marcio. Engenharia de Requisitos Intencional: Um Método de Elicitação, Modelagem e Análise de Requisitos. Rio de Janeiro, 2008. 261 p. Tese de Doutorado - Departamento de Informática, Pontifícia Universidade Católica do Rio de Janeiro.

Nos dias atuais, muito mais do que no passado, é sabido que o sucesso de projetos de software depende criticamente de requisitos. Um exemplo de GORE, que significa Engenharia de Requisitos Orientada a Metas, é o Framework i* (iestrela). Este afirma que os requisitos devem representar a intencionalidade do grande número de atores sociais, os quais são pessoas ou sistemas. Dentro desse contexto, existe ainda um vazio de métodos para cobrir o processo da elicitação de metas. Vários métodos para SMA (Sistemas Multi-Agentes) mencionam a elicitação das metas, mas eles não fornecem detalhes de como essa atividade é feita, eles tratam principalmente da modelagem de metas sem antes cuidar dos detalhes da elicitação. Em adição, modelos intencionais como exemplo o Framework i*, podem ser complexos e até mesmo incompreensíveis. Esta tese propõe um método chamado ERi*c - "Engenharia de Requisitos Intencional”, o qual primeiro provê um processo de elicitação que aplica uma abordagem "bottom-up” e simples, com perguntas que podem identificar metas concretas e metas flexíveis. Além disso, o método Eri*c propõe uma solução para reduzir o problema da "escalabilidade" de modelos $\mathrm{i}^{*}$ que inclui heurísticas para a especificação desses modelos. O método contém também uma estratégia para fazer diagnósticos de modelos $i^{*}$ de maneira a cobrir o processo de análise de requisitos.

\section{Palavras-chave}

GORE, Engenharia de Requisitos Orientada a Metas; Engenharia de Requisitos Orientada a Agentes, Elicitação de Metas, Modelo de Metas, Engenharia de Requisitos, SMA, Sistemas Multi-Agentes. 


\section{Abstract}

Oliveira, Antonio de Padua Albuquerque; Leite, Julio Cesar Sampaio do Prado (Advisor); Cysneiros, Luiz Marcio (Co-advisor). Intentional Requirements Engineering: A Method for Requirements Elicitation, Modeling, and Analysis. Rio de Janeiro, 2008. 261 p. Doctoral Thesis Computer Science Department, Pontifical Catholic University of Rio de Janeiro.

Nowadays, much more than in the past, it is known that the success of software projects depends critically on the requirements. Goal Oriented Requirements Engineering - GORE, for example i* Framework, says that requirements must represent the intentionality of a large number of social actors, which can be people or systems. Several Multi-Agent Systems (MAS) methods mention goals elicitation but they do not provide details of how this is performed, they mainly focus on goals modeling. In this context, there is still a lack of methods to cover the goal elicitation process. Only after eliciting goals, requirements engineers will be able to deal properly with goal models. Typically, this is a difficult task to carry on since requirements engineers are not familiarized with the domain from the early stages of software development. And, intentionality models, for example $\mathrm{i}^{*}$ Framework, can be complex and incompreensible. This thesis proposes a method called ERi*c - "Engenharia de Requisitos Intencional” which provides an inquire process that can identify goals and softgoals in a bottom-up and simple elicitation approach together with one

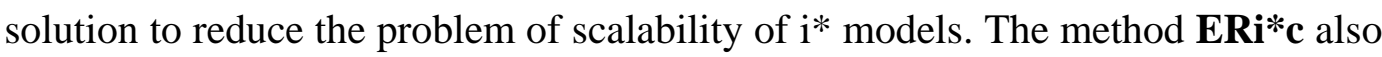
includes heuristics for modeling specification and a diagnoses aproach in order to analyze i* models.

\section{Keywords}

GORE, Goal-Oriented Requirements Engineering; Agent-Oriented Requirements Engineering, Goals Elicitation, Goals Model; Requirements Engineering; MAS, Multi-Agent Systems. 


\section{Sumário}

1 Introdução 21

$\begin{array}{lll}1.1 & \text { Motivação } & 21\end{array}$

1.2 O Objetivo da Pesquisa 22

1.3 Organização da Tese 24

2 Técnicas, Métodos e Conceitos 26

2.1 Técnicas, Métodos e Conceitos Utilizados 26

2.1.1 O Léxico Ampliado da Linguagem 26

2.1.2 A Técnica de Cenários 28

2.1.3 O Framework de Modelagem i* 29

2.2 Técnicas, Métodos e Conceitos Adaptados ou Criados 35

2.2.1 Os Conceitos de Ação Concreta e de Ação Flexível 35

2.2.2 As Estruturas Canônicas do Framework i* 36

2.2.2.1 Situações de Dependência Estratégica - SDsituations 37

2.2.2.2 Construtos de Razão Estratégica - SRconstructs 41

2.2.3 O Painel da Intencionalidade - Diagrama IP 41

2.2.3.1 As Relações entre as Metas 42

2.2.3.2 Diagrama de Classes do Diagrama IP 46

2.2.4 Diagnósticos i* (“i* Diagnoses”) 47

2.2.4.1 A Motivação para Diagnósticos i* 47

2.2.4.2 A Estratégia de Perguntas do Framework Diagnosticos i* 51

2.3 Conclusão 53

3 O Método ERi*c - Engenharia de Requisitos Intencional 54

3.1 Visão Geral do Método 54

3.2 Etapas do Método Engenharia de Requisitos Intencional - ERi*C 56

1) Elicitar as Metas dos Atores 56

1.A) Preparar LAL - Léxico Ampliado da Linguagem 57

1.B) Definir AGFL - Metas dos Agentes vindas do Léxico 58

1.B.1) Identificar os atores $\quad 58$ 
1.B.2) Extrair as metas dos atores a partir dos símbolos 59

1.B.2.a) Definir metas a partir de símbolos tipo sujeito 60

1.B.2.b) Definir metas reflexivas 62

1.B.2.c) Definir metas a partir de símbolos tipo objeto 62

1.B.2.d) Definir metas a partir de símbolos tipo verbo 64

1.B.2.e) Definir metas a partir de símbolos tipo estado 65

1.C) Refinar as metas 66

1.C.1) O Diagrama de Classes da AGFL 67

2) Identificar as Situações de Dependência Estratégica 69

2.A) Distinguir SDsituations $\quad 69$

2.B) Reconhecer Interdependências entre SDsituations 70

$\begin{array}{ll}\text { 2.C) Construir Diagrama de SDsituations } & 70\end{array}$

3) Modelar as Metas dos Atores $\quad 71$

3.A) Identificar Agentes, Posições e Papéis $\quad 72$

3.B) Criar os Painéis de Intencionalidade $\quad 73$

3.B.1) A Aplicação da Complexidade Ciclomática 75

4) Modelar a Racionalização das Metas dos Atores 75

4.A) Construir Modelos SD 76

4.A.1) Definir as dependências estratégicas $\quad 76$

4.A.2) Preparar um Modelo SD para cada SDsituation 77

$\begin{array}{ll}\text { 4.B) Construir Modelos SR } & 78\end{array}$

5) Especificar as SDsituations $\quad 81$

5.A) Descrever as Situações de Dependência Estratégica 82

6) Analisar a Racionalização das Metas dos Atores 83

6.A) Identificar estruturas canônicas $\quad 86$

6.B) Aplicar Framework de perguntas 86

6.C) Verificar as perguntas respondidas $\quad 88$

3.3 Conclusão $\quad 88$

4 Exemplos da Aplicação do Método ERi*c 90

4.1 Caso: Controle do Caixa do Restaurante 90

(1) Elicitar as Metas dos Atores 91

a) Preparar LAL - Léxico Ampliado da Linguagem 91 
b) Definir AGFL - Metas dos Agentes Vindas do Léxico 91

b.1) Definir metas a partir de símbolos tipo sujeito 92

b.2) Metas reflexivas 93

b.3) Definir metas a partir de símbolos tipo objeto 93

b.4) Definir metas a partir de símbolos tipo verbo 100

b.5) Definir metas a partir de símbolos tipo estado 106

(2) Identificar as Situações de Dependência Estratégica 111

a) Distinguir SDsituations 111

b) Reconhecer as interdependências entre as SDsituations 112

C) Construir o diagrama de SDsituations 112

(3) Modelar as Metas dos Atores 113

a) Identificar Agentes, Posições e Papéis 113

b) Criar os Painéis de Intencionalidade 113

c) Aplicação da Complexidade Ciclomática de McCabe 114

(4) Modelar a Racionalização das Metas dos Atores 116

a) Construir Modelos SD 116

b) Construir Modelos SR 116

(5) Especificar as SDsituations 121

a) Descrever as SDsituations 121

(6) Analisar os Modelos SD e SR 123

a) Identificar as estruturas canônicas 123

b) Aplicar Framewor de perguntas 123

C) Verificar as perguntas respondidas 131

4.2 Caso: Expert Committee - EC 132

(1) Elicitar as Metas dos Atores 132

a) Preparar LAL - Léxico Ampliado da Linguagem 133

b) Definir AGFL - Metas dos Agentes Vindas do Léxico 134

b.1) Definir metas a partir de símbolos tipo sujeito 134

$\begin{array}{ll}\text { b.2) Metas reflexivas } & 135\end{array}$

b.3) Definir metas a partir de símbolos tipo objeto 136

b.4) Definir metas a partir de símbolos tipo verbo 139

b.5) Definir metas a partir de símbolos tipo estado 140 
(2) Identificar as Situações de Dependência Estratégica 146

a) Distinguir SDsituations 146

b) Reconhecer as interdependências entre as SDsituations 147

c) Construir o diagrama de SDsituations 148

(3) Modelar as Metas dos Atores 149

a) Identificar Agentes Posições e Papéis 149

b) Criar os Painéis de Intencionalidade 150

c) Aplicação da Complexidade Ciclomática de McCabe 153

(4) Modelar a Racionalização das Metas dos Agentes 154

a) Construir Modelos SD 154

b) Construir Modelos SR 154

(5) Especificar as SDsituations 160

a) Descrever as SDsituations $\quad 160$

(6) Analisar os Modelos SD e SR 163

a) Identificar as estruturas canônicas 163

b) Aplicar Framewor de perguntas 163

c) Verificar as perguntas respondidas 167

4.3 Caso: Seguradora Imperial 168

(1) Elicitar as Metas dos Atores 168

a) Preparar LAL - Léxico Ampliado da Linguagem 168

b) Definir AGFL - Metas dos Agentes Vindas do Léxico 171

b.1) Definir metas a partir de símbolos tipo sujeito 171

$\begin{array}{ll}\text { b.2) Metas reflexivas } & 174\end{array}$

b.3) Definir metas a partir de símbolos tipo objeto 174

b.4) Definir metas a partir de símbolos tipo verbo 180

b.5) Definir metas a partir de símbolos tipo estado 188

(2) Identificar as Situações de Dependência Estratégica 195

a) Distinguir SDsituations 195

b) Reconhecer as interdependências entre as SDsituations 197

c) Construir o diagrama de SDsituations 197

(3) Modelar as Metas dos Atores 198

a) Identificar Agentes Posições e Papéis 198 
b) Criar os Painéis de Intencionalidade 199

(4) Modelar.a Racionalização das Metas dos Atores 201

a) Construir Modelos SD 201

b) Construir Modelos SR 201

4.4 Conclusão 202

5 A Experimentação do Método Proposto 204

5.1 A Motivação para a Experimentação 204

5.2 Definição Sucinta do Processo de Experimentação 205

5.3 Os Estudos da Experimentação 206

5.3.1 O Estudo das Competências do Método ERi*c e do Framework i* 207 1 - ETAPA DE DEFINIÇÃO 207

2 - ETAPA DE PLANEJAMENTO 209

3 - ETAPAS DE EXECUÇÃO E DE ANÁLISE 214

3.1 - Operacionalização e análise da experimentação 1

3.2 - Operacionalização e análise da experimentação 2

3.3 - Análise das Hipóteses 233

3.4 - Operacionalização e análise da experimentação 3233

5.4 Conclusão da Experimentação 238

6 Conclusão 240

6.1 Resumo e contextualização 240

6.1.1 O Método ERi*C 240

6.1.2 Avaliação dos estudos de casos 241

6.1.3 O resultado dos experimentos 242

6.2 Declaração de contribuição e comparação com outras propostas 243

6.2.1 As contribuições do Método ERi*c 243

6.2.2 Os Principais Métodos GORE 244

6.2.2.1 O que se entende por meta nos principais métodos GORE 245

6.2.2.2 GBRAM (Goal Based Requirements Analysis Method) 246

6.2.2.3 KAOS (Knowledge Acquisition in autOmated Specification) 246

6.2.2.4 $\mathrm{ERi}^{*} \mathrm{C}$ (Engenharia de Requisitos Intencional) 246

6.2.3 Métodos que possuem pontos em comum com o Método ERi*c 247 
6.3 Limitações, Trabalhos Futuros e Desdobramentos

6.3.1 Limitações do Método ERi*c 248

6.3.2 Trabalhos Futuros 248

$\begin{array}{lll}\text { 6.3.3 Desdobramentos } & 249\end{array}$

7 Referências Bibliográficas 250

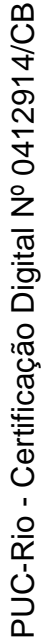




\section{Lista de Figuras}

Figura 2.1 - Os elementos do LAL representados em um diagrama de classes 27

Figura 2.2 - Os relacionamentos entre os elementos do cenário representados em um diagrama de classes 28

Figura 2.3 - Ilustração da dependência entre atores utilizada em modelos SD 30

Figura 2.4 - Meta modelo do Modelo SA - adaptado de [Leite 07] 31

Figura 2.5 - Ilustração de um Modelo AS 32

Figura 2.6 - Exemplo de um Modelo SR (parte) 33

Figura 2.7 - Ilustração das estruturas canônicas do i* 36

Figura 2.8 - As quatro variantes de interdependências lógica e temporal 38

Figura 2.9 - Diagrama de Classes da SDsituation 40

Figura 2.10 - Ilustração da relação de correlação entre as metas 43

Figura 2.11 - Ilustração das relações entre as metas em um diagrama IP 43

Figura 2.12 - Ilustração de um Painel de Intencionalidade 45

Figura 2.13 - Diagrama de classes do Painel de Intencionalidade 46

Figura 2.14 - Modelo SR (parte) - uma SDsituation e dois SRconstructs 50

Figura 2.15 - Meta Modelo do Framework i* usando o Diagrama de Classes 52

Figura 3.1 - Visão geral do método ilustrando o encadeamento das etapas 55

Figura 3.2 - Detalhamento da etapa 1 do Método ERi*C 56

Figura 3.3 - Regras gerais para definição de símbolos [C\&L - PUC-Rio] 57

Figura 3.4 - Exemplo de um símbolo do LAL do tipo sujeito 61

Figura 3.5 - Exemplo de um símbolo do LAL do tipo objeto 63

Figura 3.6 - Exemplo de um símbolo do LAL do tipo verbo 64

Figura 3.7 - Exemplo de um símbolo do LAL do tipo estado 65

Figura 3.8 -Resultado da conversão de meta tipo objeto em meta tipo sujeito 66

Figura 3.9 - Exemplo com metas concretas, metas flexíveis e dependências 67

Figura 3.10 - Diagrama de classes da AGFL 68

Figura 3.11 - Detalhamento da etapa 2 do Método ERi*C 69

Figura 3.12 - Ilustração: Exemplo do Diagrama de SDsituations 71

Figura 3.13 - Detalhamento da etapa 3 do Método ERi*C 72 
Figura 3.14 - Exemplo de Diagrama IP -

Figura 3.15 - Detalhamento da etapa 4 do Método ERi*C 76

Figura 3.16 - Regras gerais para definição de dependências estratégicas $\quad 77$

Figura 3.17 - Ilustração comparativa dos tipos de interações que

Figura 3.18 - Modelo SR (ator - ator) SDsituation: VOTAÇÃO DE CONFLITOS 80

Figura 3.19 - Modelo SR (ator - agente) SDsituation: VOTAÇÃO DE CONFLITOS 81

Figura 3.20 - Modelo SR (agente - agente)

SDsituation: VOTAÇÃO DE CONFLITOS

Figura 3.21 - Detalhamento da etapa 5 do Método ERi*c

Figura 3.22 - Exemplo da especificação da

SDsituation: ACEITAÇÃO DE PROPOSTAS

Figura 3.23 - Detalhamento da etapa 6 do Método ERi*C

Figura 3.24 - Esquema SADT: Analisar a Racionalização das Metas dos Atores 85

Figura 4.1.1 - Léxico Ampliado da Linguagem - símbolos do tipo sujeito 91

Figura 4.1.2 - Template para ações concretas de símbolos do tipo sujeito 92

Figura 4.1.3 - Léxico Ampliado da Linguagem - símbolo do tipo objeto 93

Figura 4.1.4 - Template metas vindas do LAL - símbolo do tipo objeto 94

Figura 4.1.5 - Léxico Ampliado da Linguagem - símbolos do tipo objeto 97

Figura 4.1.6 - Metas concretas vindas do LAL - símbolos do tipo objeto 99

Figura 4.1.7 - Léxico Ampliado da Linguagem - símbolo do tipo verbo 100

Figura 4.1.8 - Template metas vindas do LAL - símbolo do tipo verbo 100

Figura 4.1.9 - Metas de ações flexíveis de símbolos tipo sujeito e tipo objeto 102

Figura 4.1.10 - Léxico Ampliado da Linguagem - símbolos do tipo verbo 104

Figura 4.1.11 - Metas de ações flexíveis de símbolos tipo verbo 105

Figura 4.1.12 - Metas de ações concretas de símbolos tipo verbo 106

Figura 4.1.13 - Léxico Ampliado da Linguagem - símbolos do tipo estado 106

Figura 4.1.14 - Metas de símbolos tipo estado 107

Figura 4.1.15 - Metas convertidas em “tipo sujeito” e agrupadas por ator 108

Figura 4.1.16 - Metas concretas tipo sujeito agrupadas por ator 108

Figura 4.1.17 - Metas flexíveis agrupadas por ator 109 
Figura 4.1.18 - Metas agrupadas por ator e cronologicamente organizadas

Figura 4.1.19 - Metas concretas e flexíveis organizadas em SDsituations 111

Figura 4.1.20 - O diagrama SDsituations: Caixa do Restaurante 112

Figura 4.1.21 - Agentes, Papéis e Posições - Caixa do Restaurante 113

Figura 4.1.22 - Diagramas IP: Atendimento da Mesa e Rateio dos 10\% 113

Figura 4.1.23 - Diagramas IP: Liberação da Mesa e Fechamento da Conta 114

Figura 4.1.24 - “Controle do Caixa do Restaurante” - Diagrama IP com todas as SDsituations representadas 115

Figura 4.1.25a - Modelo SD - SDsituation Liberação da Mesa 116

Figura 4.1.25b - Modelo SR - SDsituation Liberação da Mesa 116

Figura 4.1.26a - Modelo SD - SDsituation Atendimento da Mesa 117

Figura 4.1.26b - Modelo SR - SDsituation Atendimento da Mesa 117

Figura 4.1.27a - Modelo SD - SDsituation Fechamento da Conta 118

Figura 4.1.27b - Modelo SR: SDsituation Fechamento da Conta-1 119

Figura 4.1.27c - Modelo SR: SDsituation Fechamento da Conta-2 119

Figura 4.1.27d - Modelo SR: SDsituation Fechamento da Conta - Final 120

Figura 4.1.28 - Modelo SD e Modelo SR: SDsituation Rateio dos 10 \% 120

Figura 4.1.29 - SDsituation Liberação da Mesa (especificação) 121

Figura 4.1.30 - SDsituation Atendimento da Mesa (especificação) 121

Figura 4.1.31 - SDsituation Fechamento da Conta (especificação) 122

Figura 4.1.32 - SDsituation Rateio dos 10 \% (especificação) 122

Figura 4.2.1 - Léxico Ampliado da Linguagem - símbolos do tipo sujeito 133

Figura 4.2.2 - Template para ações concretas de símbolos do tipo sujeito 135

Figura 4.2.3 - Léxico Ampliado da Linguagem - símbolo do tipo objeto 137

Figura 4.2.4 - Template metas vindas do LAL - símbolo do tipo objeto 139

Figura 4.2.5 - Léxico Ampliado da Linguagem - símbolo do tipo verbo 139

Figura 4.2.6 - Template metas vindas do LAL - símbolo do tipo verbo 140

Figura 4.2.7 - Léxico Ampliado da Linguagem - símbolos do tipo estado 140

Figura 4.2.8 - Metas de símbolos tipo estado 140

Figura 4.2.9 - Metas de ações flexíveis de símbolos tipo sujeito e tipo objeto 142

Figura 4.2.10 - Metas de ações flexíveis de símbolos tipo verbo 142

Figura 4.2.11 - Metas de ações flexíveis de símbolos tipo estado 142

Figura 4.2.12 - Metas convertidas em "tipo sujeito" e agrupadas por ator 143 
Figura 4.2.13 - Metas concretas tipo sujeito agrupadas por ator

Figura 4.2.14 - Metas flexíveis agrupadas por ator 144

Figura 4.2.15 - Metas agrupadas por ator e cronologicamente organizadas 145

Figura 4.2.16 - Metas concretas e flexíveis organizadas em SDsituations 147

Figura 4.2.17 - O diagrama de SDsituations: "Expert Committee” 148

Figura 4.2.18 - O modelo SA do Expert Committee 149

Figura 4.2.19 - Atores: Agentes, Papéis e Posições do Expert Committee 149

Figura 4.2.20a - Diagrama IP - SDsituation: Formação do Comitê 150

Figura 4.2.20b - Diagrama IP - SDsituation: Submissão de Artigos 150

Figura 4.2.20c - Diagrama IP - SDsituation: Aceitação de Propostas 151

Figura 4.2.20d - Diagrama IP - SDsituation: Revisão de Artigos 151

Figura 4.2.20e - Diagrama IP - SDsituation: Votação de Conflitos 152

Figura 4.2.20f - Diagrama IP - SDsituation: Recepção de Camera-Ready 152

Figura 4.2.21a - Modelo SD - SDsituation: Formação do Comitê 154

Figura 4.2.21b - Modelo SR - SDsituation: Formação do Comitê 154

Figura 4.2.22a - Modelo SD - SDsituation: Submissão de Artigos 155

Figura 4.2.22b - Modelo SR - SDsituation: Submissão de Artigos 155

Figura 4.2.23a - Modelo SD - SDsituation: Aceitação de Propostas 155

Figura 4.2.23b - Modelo SR - SDsituation: Aceitação de Propostas 156

Figura 4.2.24a - Modelo SD - SDsituation: Revisão de Artigos 156

Figura 4.2.24b - Modelo SR - SDsituation: Revisão de Artigos 156

Figura 4.2.25a - Modelo SD - SDsituation: Votação de Conflitos 157

Figura 4.2.25b - Modelo SR - SDsituation: Votação de Conflitos 157

Figura 4.2.26a - Modelo SD - SDsituation: Recepção de Camera-Ready 157

Figura 4.2.26b - Modelo SR - SDsituation: Recepção de Camera-Ready 158

Figura 4.2.27a - Modelo SR - Chair e Reviewer 158

Figura 4.2.27b - Modelo SR - Chair e ChairAgent 159

Figura 4.2.27c - Modelo SR - ReviewerAgent e ChairAgent 159

Figura 4.2.28a - Definição do Cenário: SDsituation Formação do Comitê 160

Figura 4.2.28b - Definição do Cenário: SDsituation Submissão de Artigos 160

Figura 4.2.28c - Definição do Cenário: SDsituation Aceitação de Propostas 161

Figura 4.2.28d - Definição do Cenário da SDsituation Revisão de Artigos 161

Figura 4.2.28e - Definição do Cenário da SDsituation Votação de Conflitos 162 
Figura 4.2.28f - Definição do Cenário da SDsituation Recepção de

Camera-Ready 162

Figura 4.3.1 - Léxico Ampliado da Linguagem - símbolos do tipo sujeito 170

Figura 4.3.2 - Template para ações concretas de símbolos do tipo sujeito 173

Figura 4.3.3 - Léxico Ampliado da Linguagem - símbolos do tipo objeto 177

Figura 4.3.4 - Template metas vindas do LAL - símbolo do tipo objeto 180

Figura 4.3.5 - Léxico Ampliado da Linguagem - símbolos do tipo verbo 182

Figura 4.3.6 - Template metas vindas do LAL - símbolo do tipo verbo 184

Figura 4.3.7 - Metas de ações flexíveis de símbolos tipo objeto e tipo sujeito 186

Figura 4.3.8 - Metas de ações flexíveis de símbolos tipo verbo 186

Figura 4.3.9 - Metas de ações concreta de símbolos tipo verbo 187

Figura 4.3.10 - Metas de ações concreta de símbolos tipo verbo 188

Figura 4.3.11 - Metas de ações flexíveis de símbolos tipo estado 189

Figura 4.3.12 - Metas concretas tipo objeto convertidas em tipo sujeito 190

Figura 4.3.13 - Metas concretas tipo sujeito agrupadas por ator 192

Figura 4.3.14 - Metas concretas tipo objeto convertidas em tipo sujeito 192

Figura 4.3.15 - Metas concretas e metas flexíveis agrupadas por ator e cronologicamente organizadas 194

Figura 4.3.16 - Metas concretas e metas flexíveis alocadas por SDsituations 196

Figura 4.3.17 - O diagrama de SDsituations: “Seguradora Imperial” 197

Figura 4.3.18 - Atores: Agentes, Papéis e Posições : “Seguradora Imperial” 198

Figura 4.3.19 - Diagramas IP “Seguradora Imperial” 199

Figura 4.3.20 - Diagrama IP com todas as SDsituations 200

Figura 4.3.21 - Modelos SD e SR: SDsituation: Efetivação do conserto

$$
\text { - agente } 201
$$

Figura 5.1 - Processo de Experimentação [Mafra 06] definido por

Wohlin et al. (2000) e estendido por Amaral (2003) 205

Figura 5.2 - Medidas de tendência do experimento 1

Figura 5.3 - Tabela da distribuição do qui-quadrado (parte) 221

Figura 5.4 - Medidas de tendência do experimento 2

Figura 6.1 - Esquema do relacionamento entre os elementos do LAL 247 


\section{Lista de Tabelas, Templates e Quadros}

Tabela 3.1 - Aplicação dos templates na definição das metas 60

Tabela 3.2 - Descrevendo os elementos das SDsituations 70

Template 3.A - Usando as ações concretas de símbolos do tipo sujeito 61

Template 3.B - Usando as ações concretas de símbolos do tipo objeto 63

Template 3.C(1) - Usando símbolos do tipo verbo 64

Quadro 3.1 - Correspondência entre o processo de diagnósticos e o PDCA 84

Quadro 3.2 - Diagnóstico de SDsituations 87

Quadro 3.3 - Diagnóstico de SRconstructs $\quad 87$

Quadro 3.4 - Quadro de Metas x Problemas $\quad 88$

Quadro 4.1 - Metas x Problemas - SDsituation: Atendimento DA Mesa 131

Quadro 4.2 - Metas x Problemas - SDsituation: ACEITAÇÃo DE PROPOSTAS 167

Quadro 5.1 - Competências do Engenheiro de Requisitos a serem avaliadas 206

Quadro 5.2 - Opções sugeridas para as competências 211

Quadro 5.3- O Questionário da Experimentação do Framework i* 214

Quadro 5.4 - Os Resultados do Questionário da Experimentação 1

Quadro 5.5- Resumo dos Resultados do Questionário da Experimentação 1218

Quadro 5.6 - Resumo do Perfil dos Participantes da Experimentação 1219

Quadro 5.7 - Consolidação do Teste Qui-quadrado da Experimentação 1224

Quadro 5.8 - O Questionário da Experimentação do Método ERi*C 225

Quadro 5.9 - Os Resultados do Questionário da Experimentação 2

Quadro 5.10 - Resumo dos Resultados Questionário da Experimentação 2227

Quadro 5.11 - Resumo do Perfil dos Participantes da Experimentação 2228

Quadro 5.12 - Consolidação do Teste Qui-quadrado da Experimentação 2231

Quadro 5.13 - Análise quantitativa das competências 232

Quadro 5.14 - O Questionário da Experimentação do “i* Diagnoses” 233

Quadro 5.15a - Mapeamento da correção do exercício SEM diagnóstico 236

Quadro 5.15b - Mapeamento da correção do exercício COM diagnóstico 236

Quadro 5.16 - Gráfico de barras da correção do exercício 237

Quadro 5.17 - Consolidação dos Resultados das Experimentações 238 


\section{Lista de Abreviaturas}

AORE - Agent-Oriented Requirements Engineering

EC - Expert Committee

ERi* ${ }^{*}$ - Engenharia de Requisitos Intencional

PUC-RIO - Pontifícia Universidade Católica do Rio de Janeiro

IEEE - Institute of Electrical and Electronics Engineers

IA - Inteligência Artificial

IP - Intentionality Panel

LES - Laboratório de Engenharia de Software

MAS - Multi-Agent System

MC - Meta concreta

MF - Meta flexível

GORE - Goal-Oriented Requirements Engineering

GQM - Goal-Question-Metric

HTML - HyperText Markup Language

RNF - Requisito não funcional

$\mathrm{RF}$ - Requisito funcional

SMA - Sistema Multi-Agentes

UERJ - Universidade do Estado do Rio de Janeiro

Udl - Universo de Informação

Web - World Wide Web 